\title{
Enriching Software Process Support by Knowledge-based Techniques*
}

\author{
Barbara Dellen, Frank Maurer, Jürgen Münch, Martin Verlage ${ }^{+}$ \\ Fachbereich Informatik, Universität Kaiserslautern, Postfach 3049, 67653 Kaiserslautern, Germany \\ \{verlage, dellen, maurer, muench\}@informatik.uni-kl.de
}

\begin{abstract}
Representations of activities dealing with the development or maintenance of software are called software process models. Process models allow for communication, reasoning, guidance, improvement, and automation. Two approaches for building, instantiating, and managing processes, namely CoMo-Kit and MVP-E, are combined to build a more powerful one. CoMo-Kit is based on AI/KE technology; it was developed for supporting complex design processes and is not specialized to software development processes. MVP-E is a process-sensitive software engineering environment for modeling and analyzing software development processes, and guides software developers. Additionally, it provides services to establish and run measurement programmes in software organizations. Because both approaches were developed completely independently major integration efforts are to be made to combine their both advantages. This paper concentrates on the resulting language concepts and their operationalization necessary for building automated process support.
\end{abstract}

\section{Introduction}

Processes are present whenever information is created, transformed, or communicated. Although we distinguish between different types of processes (e.g., business processes, decision processes, and software development processes) these types share common properties. Understanding commonalities and differences between process types is a key factor for better process support. Process support includes improved communication, detailed reasoning about process features, guiding people when performing processes, improving both processes itself and their results, and automating process steps in order to gain deterministic process behavior $[10,33]$. These purposes require explicit process representations (i.e., process models).

The variety of existing process support systems, for example process-sensitive software engineering environments or workflow management systems, corresponds to the variety of process types. In this paper we focus on software development processes and software processes. A software development process is a (sub-)set of the technical activities to build a software product. Maintenance tasks are subsumed by this phrase. A software process includes all software development processes and all organizational processes needed to drive the project (e.g., managerial processes). The addition 'software' may be omitted in this paper for the sake of brevity. Interpretation of development process models, performed by a process engine, is called enactment to emphasize the necessity of user participation for process performance [13].

Process-sensitive software engineering environments use process models to provide sophisticated support for both software developers and managerial or organizational roles [15]. Enaction updates the represented process state to keep track on the real-world process. This works quite well if the process behaves as

\footnotetext{
*This work is supported by the Deutsche Forschungsgemeinschaft as part of the Sonderforschungsbereich 501 "Development of Large Systems with generic Methods". See also the explanation in the appendix.

${ }^{+}$In alphabetical order.
} 
planned. Nevertheless, from time to time events may occur which are not considered when modeling the process. The real-world process and its model do not match any longer. Process engines, as the part of process support environments which is responsible for process enactment, should provide mechanisms for replanning a project under enaction. This addresses the problem of process evolution [24]. Without these features such an environment is not applicable in real-world projects. Unfortunately, only limited solutions do exist. ${ }^{1}$

Providing such a powerful environment is one goal of our work. Two already existing approaches from knowledge engineering and software engineering domains are synthesized. The Conceptual Model Construction Kit (CoMo-Kit) was developed for supporting complex design processes (e.g., city planning). The Multi-View Process Environment (MVP-E) supports modeling and analyzing software development processes, and guides software developers. Additionally it provides services to establish and run measurement programmes in software organizations. Both research prototypes were developed completely independently. Suitability of both approaches to real problems was demonstrated [20,28]. By studying the approaches, relating their underlying assumptions, and synthesizing them we gain a better understanding about the principles of process support environments. The aim is to provide knowledge based technology for software engineering problems. The synthesized approach should allow to prescribe development processes without hindering creativity. Necessary restrictions and rules delineate human process performance. It is intrinsic to software development processes that at any time in the project the next steps can be described precisely whereas the latter steps do not have a sharp shape. Process support environments should provide mechanisms to adjust the project plan from time to time. Moreover, capturing dependencies during product evolution allows goal-directed backtracking in order to bring the project back to the right track. Although this vision is still to be achieved, promising results of synthesizing CoMo-Kit and MVP-E already exist. They concentrate primarily on technical issues rather than providing support for project managers (e.g., resource scheduling).

As a first step of integration a careful analysis revealed commonalities and differences of CoMo-Kit and MVP-E. This was used to define requirements for process-sensitive software engineering environments from our particular perspective [37]. The integration of CoMo-Kit and MVP-E requires the recognition of similar concepts. It is interesting that on the one hand the concepts of CoMo-Kit are a subset of those present in MVP-L; this is a hint that both approaches contain concepts that are intrinsic to processes in general. On the other hand, the functionality of the CoMo-Kit process engine exceeds MVP-S by alternating modeling, planning, and enaction and supporting change processes.

The next two steps of integration, the definition of a common process representation schema or language and the implementation of a prototypical process engine, are discussed in this paper. The core of the new language, which is called Modeling Language and Operational Support for Software Processes (MILOS), is enactable a common process engines. Special attention is paid to the traceability of the requirements. The way of collaboration between CoMo-Kit and MVP-E is illustrated by use of a scenario which describes replanning a process under enactment:

The scenario embodies a model of a standard implementation process, as for example described in [18]. A module's design is complete and source code is to be created. In parallel test data is to be derived either by analyzing the code (in the case of a later structural or white-box testing) or by eliciting the data out of the requirements document (in the case of a later functional or black-box test). The choice of selecting the first or second alternative of test data derivation depends on the module's control flow complexity. If complexity is high then structural testing is applied else functional testing should be selected. Because the module's complexity is not known prior to its design or implementation (depending on the complexity measure) there can't be assigned resources to the data derivation process. As soon as the value is measured, project management instantiates the corresponding process and schedules a developer responsible for it. Please note that although this could be described as a simple if-then-else situation in a process script, the task for

1.“...there is much further research work to be carried out in the area of software process evolution. Understanding and managing software process evolution seems to be one of the most difficult challenges that the software engineering community is facing today." ([24], p. 1126) 
a process management system is much more difficult. Planning makes statements - sometimes in a vague manner - about future objects. The more the project proceeds the more concrete such statements could be made. In the above example, either derivation of test data for functional or structural testing is allowed to be instantiated. Otherwise resources for both processes are scheduled. Other situations to be managed by the process engine must be a refinement of already instantiated processes (e.g., insert a refinement of derivation of test data for functional testing which includes equivalence or boundary analysis) and retracting decisions already made (e.g., the system's design is modified and the module is split into two modules).

We understand the work presented in this paper as an example of how knowledge-based techniques can be used to address one of the major problems in the area of automated support for managing software development processes.

The paper is organized as follows: The concepts implemented in MVP-E and CoMo-Kit are surveyed in Sections 2 and 3. Section 4 identifies requirements for process support environments. They are derived from a comparison of both approaches in [37]. The integration is described in the Sections 5-8: Section 5 explains the integrated system architecture, Section 6 discusses concepts of the integrated language, Section 7 gives an example, and Section 8 outlines the operationalization of the language concepts. In Section 9 we compare our findings to related research work. Finally, Section 10 summarizes the paper.

\section{MVP-E}

The MVP project aims at support for management of software development processes from a software engineering perspective. Properties of such processes are, for example:

- Many people are involved in a project and perform many different types of processes

- The processes last long, sometimes several months or years.

- Not all process steps are known in advance when planning the project.

- Erroneous performance or bad process models require repeated performance, probably also of other processes than the failed one.

The MVP project began at the University of Maryland and continues at Universität Kaiserslautern, Germany. Its goal is to provide MVP-E, an environment as an instance of the ideas developed in the TAME project [6]. Special attention is paid to measurement activities which are essential when the environment should be used by all roles of the project and the organization [23]. MVP-E supports modeling, planning, simulation, enactment, measurement, recording, and packaging of software engineering processes. The main idea is to split descriptions of software development processes into views. Every view is generally defined as a projection of a process model that focuses on selected features of the process [36].

The language MVP-L is used to describe development processes. It distinguishes between processes, products, resources, and their attributes which correspond to measurable qualities of the objects; processes, products, and resources are instantiated with respect to types and related by a project plan [8]. A second notation is used to represent GQM trees which are specifications of measurement goals from a specific viewpoint [5]. The discussion of GQM is beyond the scope of this paper. MVP-L has been evaluated in several industrial settings (e.g., [20]). The case studies' feedback became input for evolution of MVP-L. Table 1 summarizes the main concepts of MVP-L.

Figure 1 shows an excerpt of an MVP-L example from the scenario above. It stems from a formalized standard implementation process defined in the IEEE Standard 1047-1991 [18]. Since the implementation process can be seen as a typical process in a software life cycle, it is used throughout the whole paper to demonstrate the modeling styles of the different approaches. For the purpose of a comprehensible illustration, minor differences among the process descriptions in the standard and the adapted examples were accepted. 


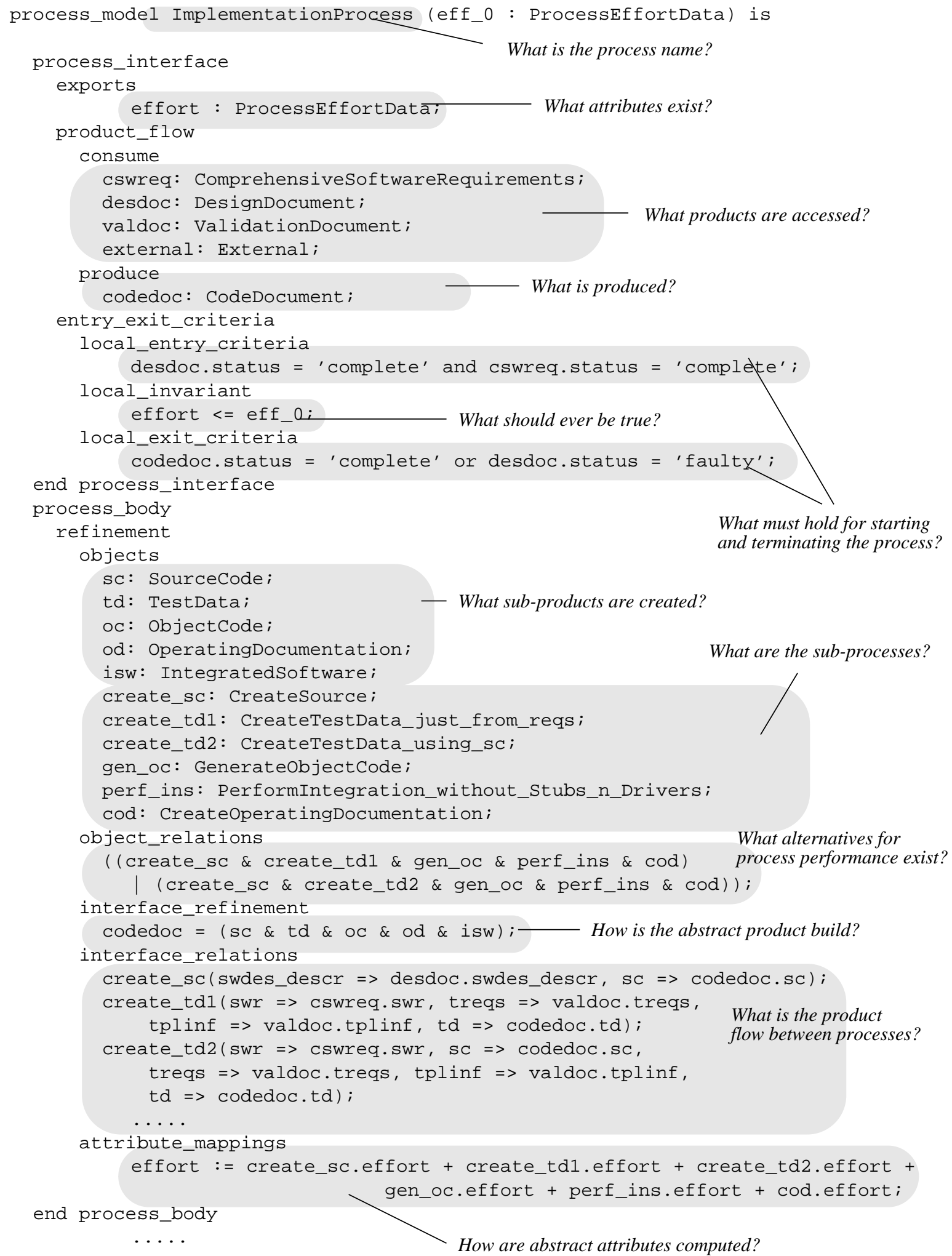

Fig. 1: MVP-L example of an implementation process with two alternatives

MVP-E's process engine MVP-S uses a project plan and process models (types) to build its own representation of a real-world project [23]. The process engine is used to manage project data and to guide developers in their work. Processes can be enacted if they are enabled (i.e., their entry criteria are true). The agents, represented as resources assigned to processes, are responsible for achieving the goals specified in the exit criteria without invalidating the process invariants. Throughout enactment, measurement data are taken which are used by all project roles (e.g., testers, managers) to reason about the project and to trigger actions. The following assumptions (labeled as AMi) were made during the evolution of the MVP-E 


\begin{tabular}{|l|l|}
\hline Concept & Explanation \\
\hline \hline Process & Activities which create, modify, or use a product. \\
\hline Product & Software artifact describing the system to be delivered. \\
\hline Resource & Human agent or tool. \\
\hline Attribute & Measurable characteristic of a process, product, or resource. \\
\hline $\begin{array}{l}\text { Criteria (entry, invariant, } \\
\text { exit) }\end{array}$ & Expression which must be true when starting, enacting, or terminating a process respectively. \\
\hline Refinement & Breaking down the structure of a process, product, or resource into less complex parts. \\
\hline Instantiation & $\begin{array}{l}\text { Creating an instance of a process type, product type, or resource type and providing actual } \\
\text { parameters. }\end{array}$ \\
\hline Product Flow & $\begin{array}{l}\text { Relationship between processes and products. It is distinguished between reading, writing, } \\
\text { and modifying access. }\end{array}$ \\
\hline Resource Allocation & Assigning personnel to processes in order to perform the processes. \\
\hline $\begin{array}{l}\text { Process Model, Product } \\
\text { Model, Resource Model }\end{array}$ & $\begin{array}{l}\text { Type descriptions of processes, products, and resources respectively. The name Model might } \\
\text { be misleading in this context, because their instances are models of real-world processes, } \\
\text { too. }\end{array}$ \\
\hline
\end{tabular}

\section{Table 1: Concepts of MVP-L}

approach and are made explicit to allow the comparison of the underlying motivations and goals of the two different approaches later on:

AM1: The concepts implemented in MVP-L are suited to express a single view on a particular software process and are made explicit to allow to compare the goals and motivation of different approaches.

AM2: Attributes of processes, products, and resources are sufficient for all measurement purposes.

AM3: Developers are guided by enacting understandable process models.

AM4: All project roles are supported by using a common representation of a project. The roles get own views on the project which are tailored to their specific needs.

AM5: Products are represented without storing their contents. Direct access to the product is not supported. Only product models are accessed.

AM6: The steps of modeling, planning and enaction are sequential. Project plans remain unchanged over the project lifetime.

AM7: Planning provides parameters which adapt process models to different contexts.

AM8: Plan deviations are not considered. No support for modifying the project's state is provided.

Several other approaches for supporting software development processes have been developed [1, 10, 33]. In general, we can distinguish between languages for modeling fine-grain processes (i.e., used to integrate tools, hence being similar to programming languages) and coarse-grain processes (i.e., used to guide software developers and to coordinate their tasks, hence being similar to specification languages). Currently, the community focuses on the latter kind of processes. An example is the evolution of the Marvel Strategy Language, which was first designed for use in a single-user system and now supports distributed teams [7]. MVP-L belongs to the second category of languages. Another classification of software process languages can be made with respect to their level of abstraction. Granularity ranges from abstract levels with rich semantics of the concepts (e.g., MVP-L) down to detailed levels which provide powerful mechanisms to build one's own process building blocks (e.g., APPL/A [34]).

Not all languages cover an equal set of aspects of software development processes. Mostly, the languages provide a solution for a particular problem and support only a limited set of roles [32]. For example, the language SLANG focuses on capturing the dynamic aspects of a process [3]. MVP-L addresses process 
interfaces, rule-based specification of development processes and measurement aspects. Quality considerations have become more and more important within software development. However measurement aspects have not been taken into account in most of the process support environments. Approaches to support measurement activities within software development already exist [22]. MVP-E is an environment which includes measurement support.

\section{CoMo-Kit}

The CoMo-Kit project at Universität Kaiserslautern aims at support for planning, enacting, and controlling complex workflows in design processes from a knowledge engineering perspective [25]. Properties of such workflows are:

- They are too complex to be planned in detail before enactment starts. Results of activities are needed to plan later steps. Planning and enacting must alternate during the whole project.

- Decisions are made during enactment which rely on assumptions that can be found invalid afterwards. When old decisions change, users must be supported in reacting to the new situation (i.e., backtracking must be supported).

The CoMo-Kit system consists of a tool which allows to model complex workflows and a workflow management system, the CoMo-Kit Scheduler, which enacts and controls the modeled workflows. To describe complex design processes, the CoMo-Kit methodology uses four basic concepts: tasks, methods, concepts, and agents. Table 2 contains an overview and short descriptions of the terms. Using these concepts generic software process models and concrete project plans can be described. The next question to be answered is how to enact these plan.

The enactment of design processes is supported by a flexible workflow management system, the CoMo-Kit Scheduler. In Table 3 concepts of the Scheduler are explained. The main features of the Scheduler are: ${ }^{1}$

- The Scheduler allows alternate planning and enactment of development processes.

- Based on the information flow between tasks, causal dependencies are acquired during enactment. The underlying assumption is that the inputs of a task influence the outputs. For every task a set of logical implications is created; the implications relate the assignment of values (i.e., products) to the input parameters of a task with the assignment of values to the output parameters. Whenever the assignment of an input parameter becomes invalid, the assignments of the output parameters become invalid too. The causal dependencies improve the traceability of design decisions and support the users in reacting to changes.

- Additionally, the scheduler manages dependencies extractable from the task decomposition. Whenever a task becomes invalid because of a replanning of the project, the Scheduler notifies team members working on subtasks that they can stop working on them.

- To handle dependencies efficiently, reason maintenance techniques are used [30,31] and extended. The Scheduler uses the acquired justification structures to support dependency-directed backtracking.

The following assumptions (labeled as $\mathbf{A C i}$ ) were made during the evolution of the CoMo-Kit approach:

AC1: Modeling, planning, and enacting design processes cannot be separated. They have to alternate.

AC2: Project plans have to be modified and refined during project lifetime.

1.The techniques which are used to get these features are beyond the scope of this paper. For a detailed description see $[27,26]$. For a description from a software engineering point of view see [11]. 


\begin{tabular}{|l|l|}
\hline Concept & Explanation \\
\hline \hline Task (Process) & A description of the goal which should be reached by an activity. \\
\hline Input Variables & Information which is needed to work on a task. \\
\hline Output Variables & Information which is the outcome of working on a task. \\
\hline Method & $\begin{array}{l}\text { A description of how a task's goal can be reached. For every task a set of alternative methods can } \\
\text { be described. }\end{array}$ \\
\hline Atomic Method & Atomic methods assign values to the output variables of the related task. \\
\hline Complex Method & Complex methods decompose a task into subtasks. \\
\hline Information Flow & $\begin{array}{l}\text { A complex method is described by an information flow graph which consists of (sub-) tasks and } \\
\text { variables. The information graph shows the input/output relations of tasks. }\end{array}$ \\
\hline Concept Class & $\begin{array}{l}\text { A description of the structure of the information (product structure) which is produced during } \\
\text { enactment. }\end{array}$ \\
\hline Slot & Stores part of product information. \\
\hline Concept Instance & $\begin{array}{l}\text { Concrete information, for instance a product (such as requirements document) which is outcome } \\
\text { of using a method to solve a task. }\end{array}$ \\
\hline Agent & An actor who works on tasks. Agents apply methods to solve tasks. \\
\hline
\end{tabular}

Table 2: The CoMo-Kit modeling framework

AC3: The Scheduler manages the state of the project. Therefore, it should have access to all products and manage them.

AC4: Users must be supported in keeping track about where the result of their work is used and what information they need in producing their results.

AC5: Changing old decisions is inherently needed in complex projects.

AC6: Causal dependencies are the basis for an active notification mechanism which informs users about relevant changes in the project state.

AC7: Only processes performed by individuals are modeled in order to keep track of dependencies.

AC8: The same formalism can be used to describe generic process models and concrete project plans.

\begin{tabular}{|l|l|}
\hline Concept & Explanation \\
\hline \hline Decision & To solve a task (i.e., to reach the goal) an agent has to decide which method should be applied. \\
\hline Assignment & Applying atomic methods to tasks results in the assignment of values to the output variables of tasks. \\
\hline Task Decomposition & $\begin{array}{l}\text { Applying complex methods to tasks results in a set of subtasks which are included into an agenda. } \\
\text { This agenda stores a list of all tasks which must be solved to finish the project. }\end{array}$ \\
\hline Dependency & $\begin{array}{l}\text { From the information flow a set of causal dependencies is derived. We assume that there is a causal } \\
\text { dependency from the inputs of a task to its outputs. The Scheduler manages these dependencies. }\end{array}$ \\
\hline Decision Retraction & $\begin{array}{l}\text { During project enactment decisions can be found erroneous which results in an inconsistent project } \\
\text { state. Then, at least one decision must be retracted and replaced by an alternative. }\end{array}$ \\
\hline
\end{tabular}

Table 3: Concepts of the CoMo-Kit Scheduler

Figure 2 shows a part of the process decomposition from our scenario modeled in CoMo-Kit. The information flow within the method "Implementation with Structural Testing" is shown in Figure 3.

Several other approaches for supporting workflows have been developed. In [16] an overview on current workflow management techniques is given. The authors show that current technology assumes that the process model is defined before process enactment. The CoMo-Kit approach was developed for application domains where planning and enactment alternate, for example design processes (e.g., city planning). Some workflow management systems support object-oriented data structures. For instance in [29] an innovative approach is described which integrates Petri nets with semantic data models. CoMo-Kit also supports object-oriented data structures. Further, CoMo-Kit supports reacting to decision changes. 

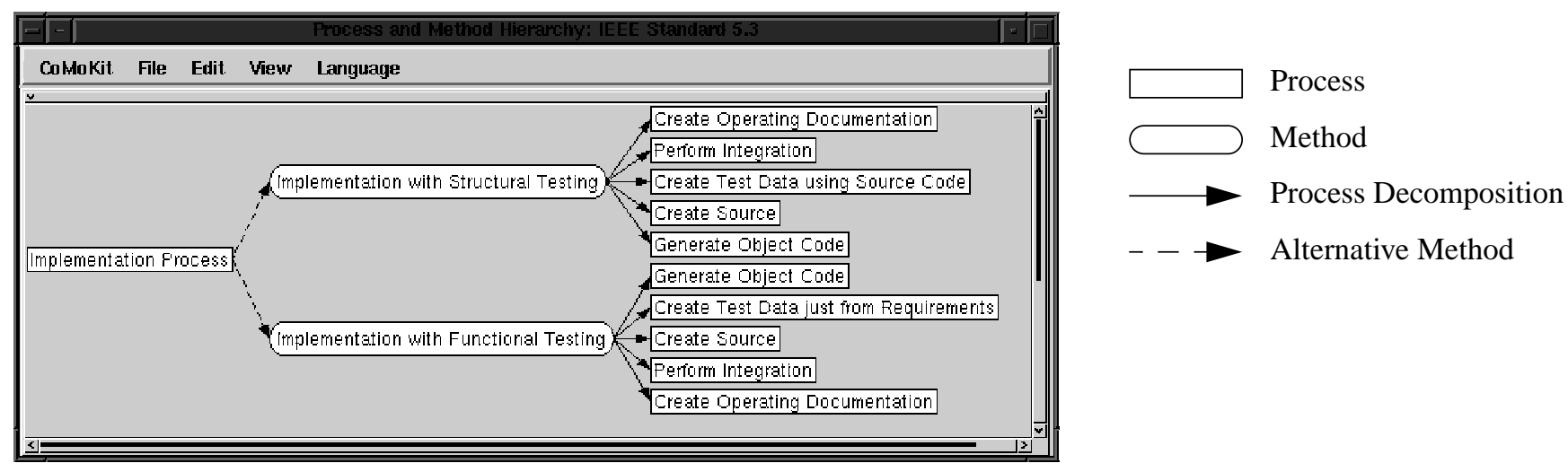

Fig. 2: Process decomposition in CoMo-Kit
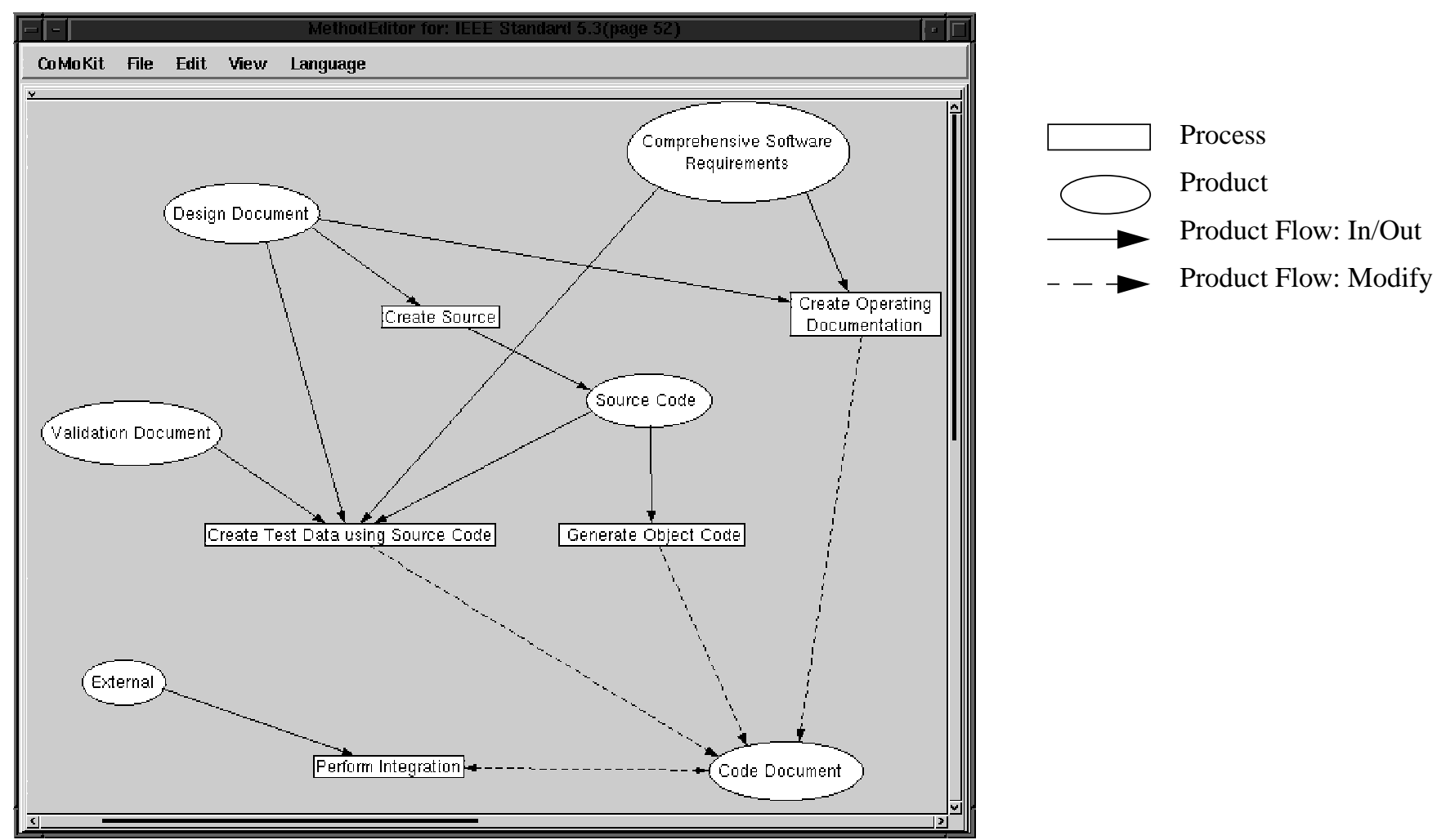

Fig. 3: Product flow in CoMo-Kit

\section{Requirements}

Section 2 and Section 3 presented assumptions which guided the evolution of two process support approaches. The assumptions can be regarded as requirements for a process-sensitive software engineering environment. The following list of requirements for process support environments reformulates the assumptions made in both approaches. Incompatibilities caused by different assumptions in the two approaches are removed (as shown in [37]), so that the requirements represent a unified view. For each requirement (labeled as $\mathbf{R i}$ ) the related assumption(s) (AMi or ACi) are given. The set of requirements is not to be understood as an exhaustive list of features a process-sensitive software engineering environment should have. The requirements were defined from the perspectives of both systems CoMo-Kit and MVP-E, nevertheless they are mandatory for each environment to support real development processes. Some aspects are not discussed in this paper (e.g., resource scheduling, time planning, budgeting).

R1: The main concepts of software development processes should be provided. (AM1, AC7, AC8) Algorithms for planning, analyses, or enactment need to know the semantics of basic entities (e.g., processes, 
products, product flow). It is important to tailor the concepts' representation to human and machine understanding.

R2: Organizational processes should be supported. (AM4) Specific interfaces to organizational processes are required. Further development in the area of multiple views on a project representation is needed in order to gain role-specific representations [36].

R3: Both short-term planning (detailed level) and long-term-planning (abstract level) are supported (AM7, AC1). It is desirable to plan activities early in a project, but it is not practical in every case. Mechanisms are needed on the one hand for planning abstract and general processes and on the other hand for planning concrete and state-dependent ones.

R4: Allow for alternating modeling, planning and enaction. (AM6, AC1, AC2) Information for planning is incomplete when launching the project. Required information may be produced during the project. Later planning steps refine the models or the original plan.

R5: Document decisions. (AC4, AC6) Many decisions about products and processes need to be documented [34]. The decisions explain how products evolve and processes are performed. The decisions are used to recognize deviations and to signal inconsistencies to the system. Dependencies between decisions can be used for backtracking and also for reasoning about products [11].

R6: Reactions on changing decisions. (AC5) When an invalid state is reached after retracting a decision, backtracking should be performed in order to reach a branch where an alternative can be chosen.

R7: Manage the products whenever possible. (AM5, AC3) Inconsistencies between the real-world project and the representation managed by the environment must be avoided.

R8: Support measurement activities. (AM2) Quantitative data from processes and products are needed in order to develop software systematically [33]. Measurement, evaluation, and storage of data is needed.

R9: Software developers need to be guided. (AM3, AC4) Process models explain what activities to perform next and what their goals are.

R10: Software developers' tasks need to be coordinated. (AM4, AC6) Process models are used to relate tasks of software developers. In the case a result becomes invalid, coordination means notifying others to interrupt their work.

R11: Execution of process fragments. Process programs are used to automate process steps. It must be ensured that the dependencies established during a tool invocation are captured and documented in the environment's repository.

The first ten requirements describe features of either CoMo-Kit or MVP-E. Automatic execution of process programs is necessary, but a straightforward to realize next step. Commercially available process-sensitive software engineering environments (e.g. Process Weaver [14]) have already demonstrated the suitability of a shell-like language for the definition of process programs. Building a system which fulfills all mentioned requirements is the challenge of integrating CoMo-Kit and MVP-E. Nevertheless, there are still open problems which are not tackled by the synthesis of both approaches:

P1: State Manipulation. When a project deviates from the plan it might be necessary to shift the whole project state by a "brute-force" manipulation of state variables instead of performing backtracking. The problem is how to modify the models according to the new state of the real-world project and to preserve existing dependencies.

P2: Type-Instance Correspondence. Direct manipulation of instances results in a project trace which cannot be described by the plan's process types. This is also true when types of active processes are modified. Mechanisms are needed to establish correspondence between instances and types.

P3: Existing Products and Measurement Data. Backtracking and choosing an alternative should not mean throwing away the results already produced. Mechanisms for reuse within a project are needed. At the 
moment it is not clear how to handle measurement data in the case of backtracking. For example, effort data should be kept but statements about products (e.g., subjective classification of complexity) might become invalid.

Although the requirements listed in this section present a unified view of both approaches this does not mean that a particular user has to manage such a tool in its entire complexity. Some features might be interesting for only some roles (e.g., a project planner is interested in R4 but not in R9) and some functionality should be kept completely away from users (e.g., R10).

\section{The Integrated System Architecture}

Within the CoMo-Kit project of the University of Kaiserslautern techniques, methods, and systems were developed which support planning and enacting complex distributed cooperative design processes $[28,12]$. This system is the basis for the prototypical implementation of an integrated system, called the MILOS environment, which fulfills requirements R1-R11.

Figure 4 shows the system architecture of MILOS. It consists of three main parts:

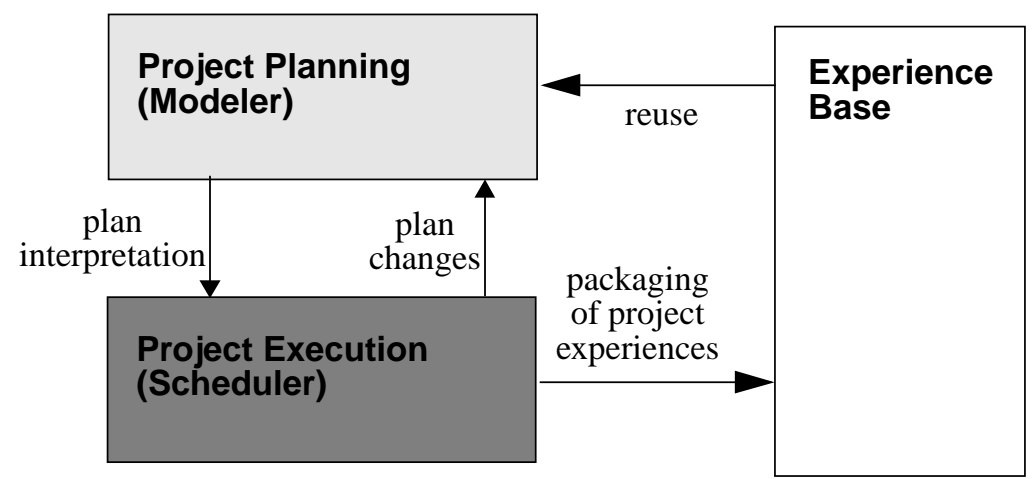

Fig. 4: The Architecture of the MILOS System

- The Modeler allows to plan and replan a project.

- The Scheduler supports the enactment of a project and manages the information produced.

- The Experience Base stores generic (reusable) project plans, products etc.

The current project plan is used by the MILOS Scheduler which supports project enactment. Several clients are implemented to work on subtasks of the process. The Scheduler may actively send messages to the clients, i.e. the system extends the typical client server architecture towards an agent-oriented structure.

Building the experience base is a future topic of our work and the results are too preliminary to be described here. We will follow the line of work described in [4]. This paper concentrates on the concepts behind the Modeler and the Scheduler.

\section{MILOS: A Language for Project Planning}

Project planning means developing a model of how the project should be performed. At the beginning of a new project, a first step creates an initial project plan. This plan contains descriptions of process types, definitions of products to be created and a list of the team members involved in the development process. For large-scale projects, a detailed plan cannot be developed before the enactment starts but planning and execution steps must be alternated (R4): Starting with the initial plan the first processes are enacted. Based on the results, the plan is refined and/or extended (see scenario in Section 1).

To model cooperative development processes, our approach uses four basic notions (fulfilling R1): Process Types, Methods, Products and Resources. In the following, these terms are defined as far as it is necessary 
to understand this paper omitting syntactical details of our project planning language. Later on in Section 7, our modeling language MILOS is illustrated by an example.

\section{Process Types}

A process type describes an activity which must be carried out during process enactment to reach the goals of the project. The description of a process type consists of several parts:

Goal. A (textual, informal) description of the goal of the activity which will be accessed by the process performers during enactment. The goal guides developers by explicitly stating what should be achieved by enacting a process.

Product Parameters. A product parameter will store input, output and modified products during enactment. Inputs are consumed during process enactment to produce the outputs of the activity. Parameters which can be changed during enactment are stored in the modified parameter list. In the project plan, we are only able to state which type of information is used or must be produced. For every input the flags ${ }^{1}$ mentioned in Table 4 are defined. Outputs of a task may be optional or required.

\begin{tabular}{|l|l|}
\hline Flag Name & Meaning \\
\hline \hline necessary for planning & $\begin{array}{l}\text { The input must be available before the planning } \\
\text { of the process starts. Planning here means } \\
\text { defining a method or choosing on of the pre- } \\
\text { defined methods. }\end{array}$ \\
\hline necessary for execution & $\begin{array}{l}\text { The input is not needed for planning but it must } \\
\text { be available before the execution starts. Execu- } \\
\text { tion here means applying the method. }\end{array}$ \\
\hline optional & $\begin{array}{l}\text { The input is neither needed for planning nor for } \\
\text { execution (but it may be helpful to have). }\end{array}$ \\
\hline
\end{tabular}

Table 4: Parameter Flags

Context information. A list of references to information which is not changed by the process enactment (e.g. a file containing the coding standards of the company or a reference to manuals).

Precondition. A formal, boolean condition using process and product attributes which must hold before the process enactment may start. Preconditions are, for example, used to check if the inputs fulfil a given requirements.

Invariant. A formal, boolean condition using process and product attributes which must hold during process enactment. An invariant, for example, may check that the time for the process stays below a given limit.

Postcondition. A formal, boolean condition using process and product attributes which must be true after the process enactment has finished. Postconditions are, for example, used to check if the output of a task has a desired quality.

Agent bindings. For every process type, the planner may state criteria which must be fulfilled by agents to be allowed to work on the process during enactment. For example, an agent must have skills in Smalltalk-80 programming and belong to department ZFE 153. We distinguish two types of agent bindings: Process performer and process supporter. The performer is responsible for the execution of the process and has to produce the outputs. He may be supported by (several) other agents.

Attributes. An attribute describes a feature of the process type, e.g. the time needed for its enactment.

Methods. A list of alternative courses of action which can be used to reach the goal. The process type describes what shall be done, methods describe how it can be done.

1.The flags are mutually exclusive. 


\section{Products}

To model products which are created in the course of project enactment, a standard object-centered approach is used. As usual, we distinguish between types and instances (for sake of brevity, we will use the term "product" for "product instance"). Types define a set of slots to structure the product. Every slot is associated with its type. Types may be basic types (e.g., STRING, REAL, ...) or defined and aggregated by the modeler. During process enactment we represent product instances as values which are assigned to parameters. The type of a parameter is specified by a product class. Using other product types as type of a slot creates complex object structures. A slot contains a part of the product whereas an attribute describes a feature of it, e.g. the complexity of a module.

\section{Methods}

A method describes how the goal of a process can be achieved. For every process type, the project plan may contain a set of (predefined) alternative methods ${ }^{1}$.

Methods are executed by agents (see below). Not every agent who may be responsible for a process may have the abilities to use every method (For example, an implementation process may be enacted by the methods "Implement in C++" or "Implement in Smalltalk"). Therefore, we allow to describe additional agent bindings for every method.

We distinguish between atomic and complex (or composed) methods.

The application of atomic methods assign products to parameters. Process scripts describe how a given task can be solved by a human. Process programs are specified in a formal language so that computers can solve a task automatically without human interaction. For an atomic method it is possible to specify what (software engineering) tools are used during enactment.

Complex methods describe the decomposition of a process into several subprocesses. For every (sub)process type, its cardinality is given which determines how many instances of this process shall be created during enactment. It maps the output parameters of one subprocess to the input of another resulting in a horizontal product flow between subprocesses (product interface relations). Additionally, it maps the parameters of the superprocess to parameters of the subprocesses resulting in a vertical product flow between a process and its parts (product mapping). In Figure 5 the relation between a process type, one method and the subprocesses is illustrated.

Finally, a method describes how attributes of the subprocesses can be used to compute attributes of the superprocess (attribute mapping).

\section{Resources: Agents \& Tools}

Resources are used for project planning and process enactment and execution. Agents are active entities which use (passive) tools for their work.

Processes are either performed by actors (= human agents) or by machines. The first case is called ,enactment", the second ,execution“".

For every process type, the project plan defines the properties an agent must have to work on it. Further, our system stores information about the properties of every agent. For actors, we distinguish three kinds of properties: qualifications (q), roles (r), and organization (o).

Example: In a project plan, it is defined that the process type "implement user interface" should be executed by an actor which has skills in using the Visualworks Interface Builder (q), is a programmer (r), and

1.For example, reusable methods may be extracted from old project traces and stored in the experience base. Then they can be incorporated into the current project plan. 


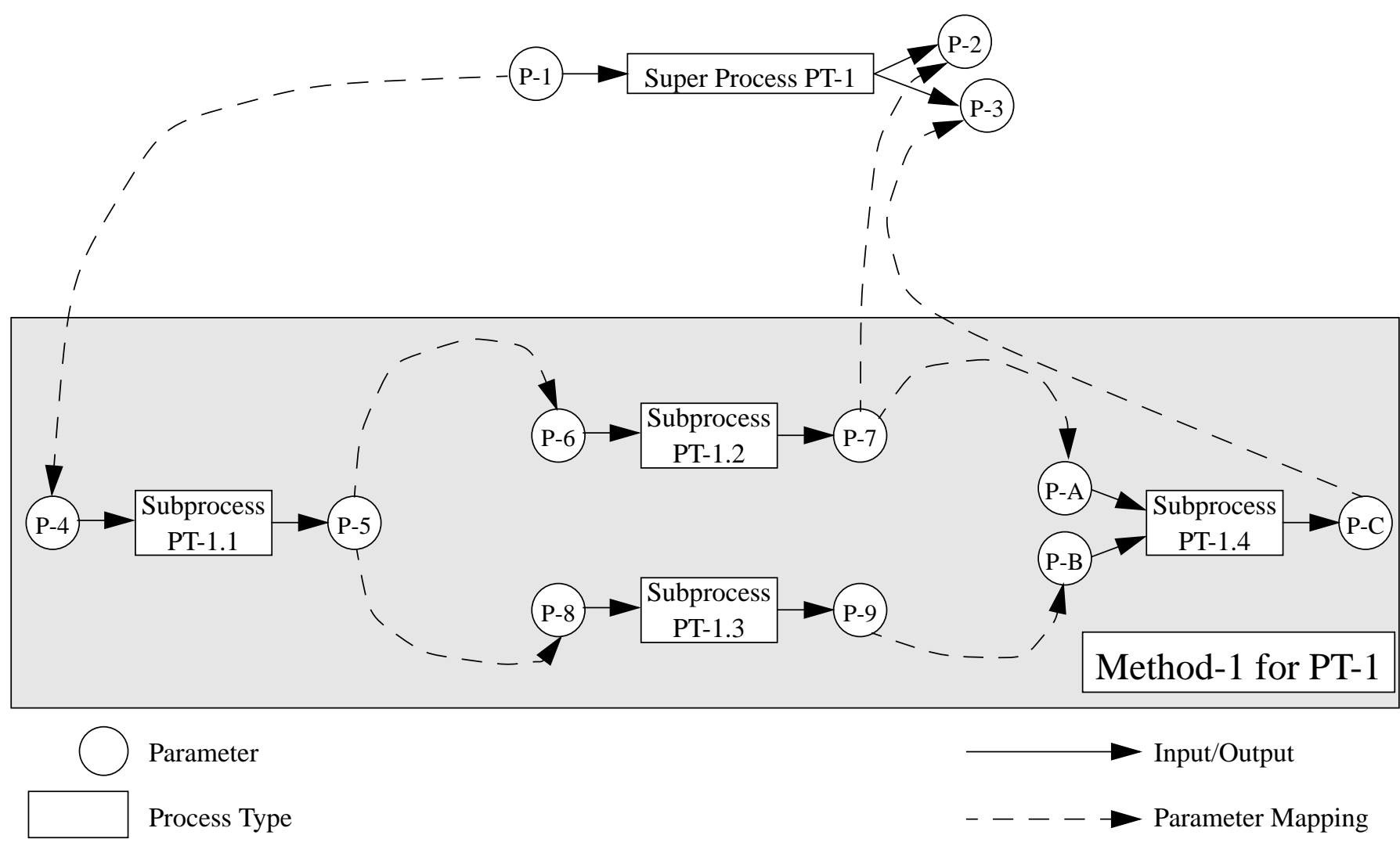

Fig. 5: Vertical and horizontal mapping of parameters

works

in department ZFE 153 (o).

During task execution, our system compares the required properties of a task with the properties an agent possesses. This allows to compute the set of agents which is able to solve the task.

Having sketched our language for project planning (which is basically an extension of MVP-L with methods and object-oriented data modelling facilities), we now will give an example of its use before we explain how the enactment of plans is supported.

\section{A MILOS Example}

Figure 6 shows the reference process as part of a MILOS adaption of the scenario mentioned above. The intended representation for modeling in MILOS is graphical. For the purpose of a compact description the example here is given in a textual representation.

To perform the implementation process two alternative methods are offered. Depending on a decision which can be influenced by measured data one of these methods can be applied, e. g., implementation with structural testing (see Fig. 7). This method is complex and it refines the implementation process into several subprocesses.

\section{Supporting Planning and Enactment: The MILOS Scheduler}

Based on the requirements in Section 4 we implemented a workflow engine, the MILOS Scheduler. Its main features are:

- It provides the people involved in the project with relevant plan, process and context information to optimize their work and to reduce the information procurement time. This includes the distribution of the processes to the appropriate employees and making available information they need for enactment such as products and the process goal descriptions. 


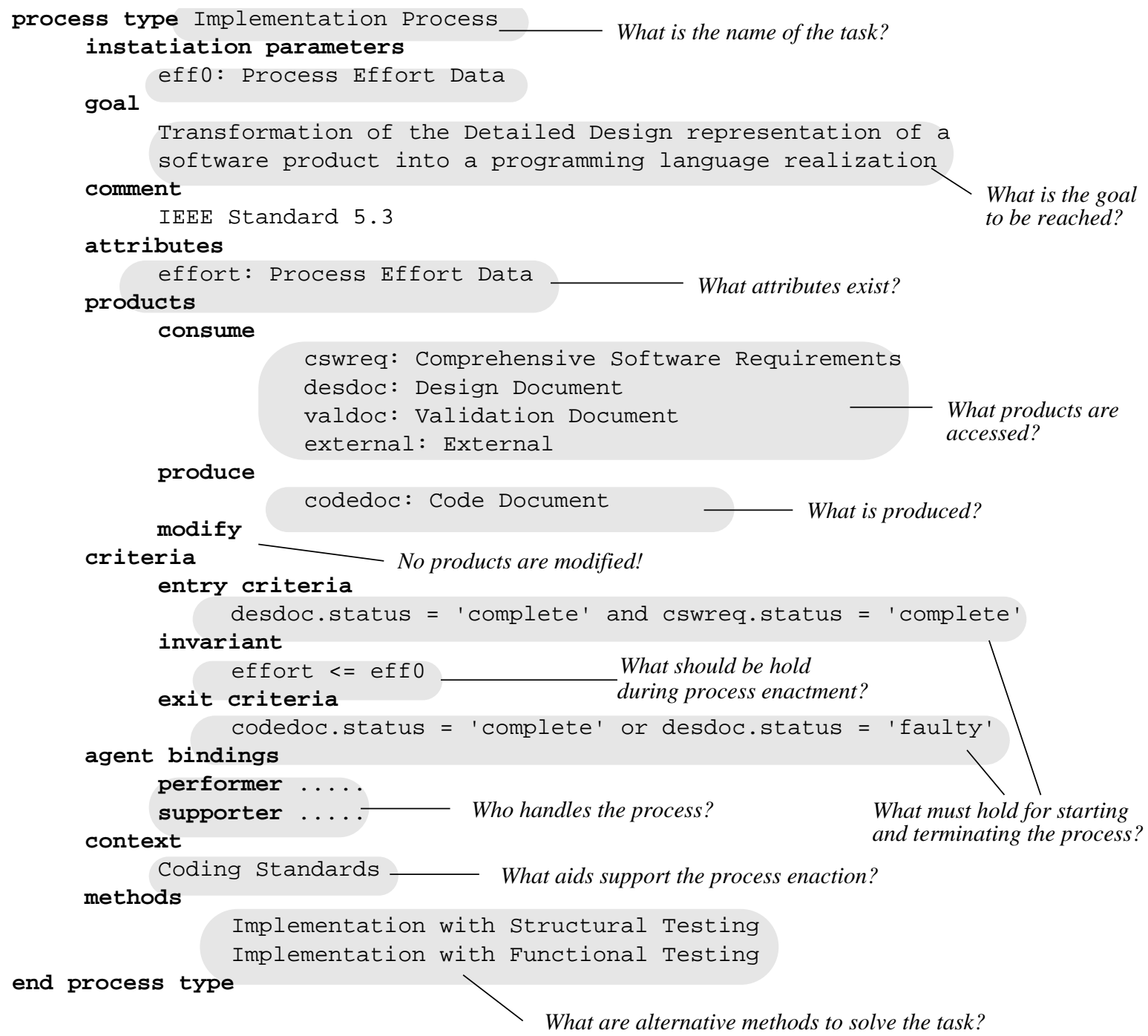

What are alternative methods to solve the task? and terminating the process?

Fig. 6: Excerpt of a MILOS example: Implementation process with two alternatives

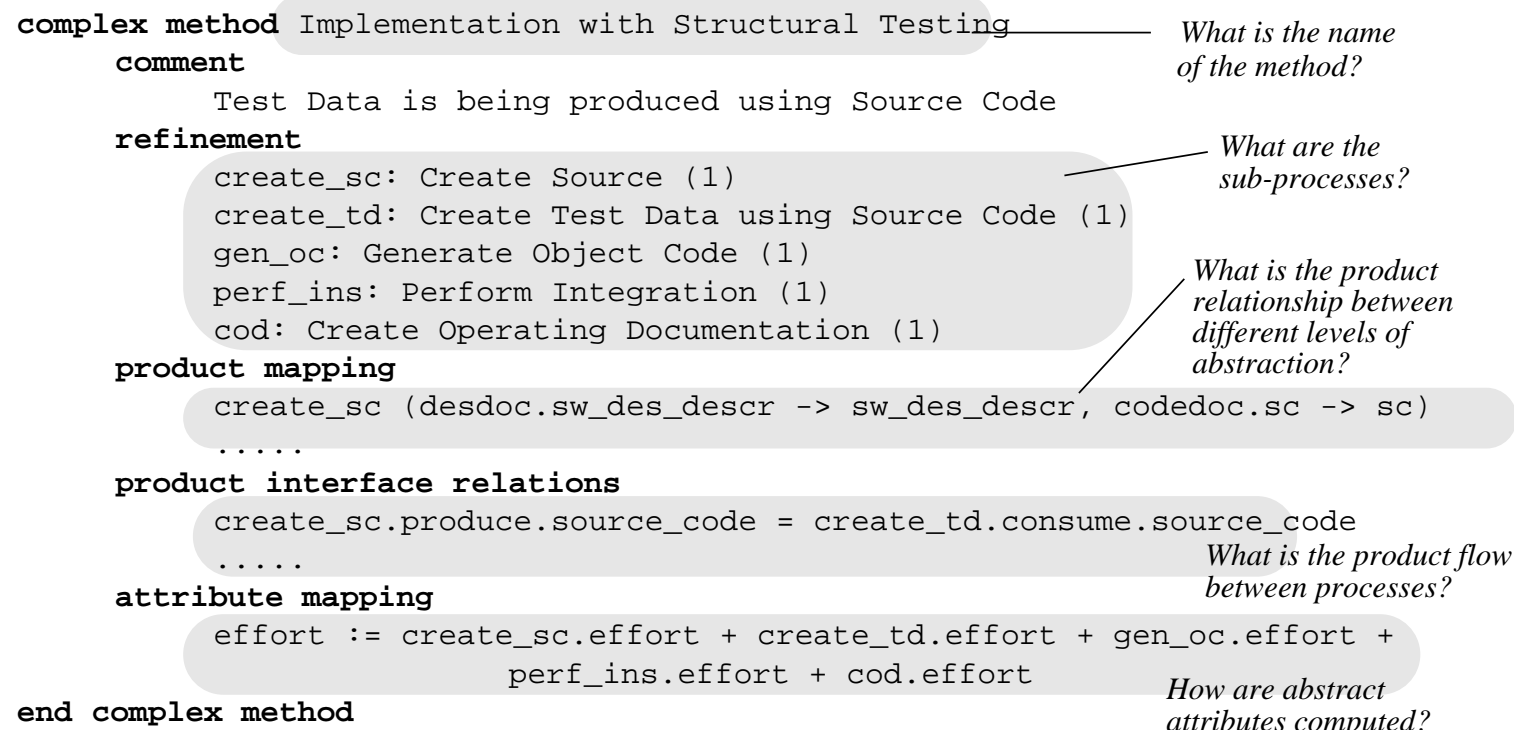
create_sc (desdoc.sw_des_descr $\rightarrow$ sw_des_descr, codedoc.sc $\rightarrow$ sc)

What is the name of the method?

What are the sub-processes?

What is the product relationship between different levels of abstraction? 
- It reduces coordination effort of each team member, by notifying the process performer on events (for example plan changes and modified products) he is affected from.

- It allows to intervene in the planning process during process enactment.

- It allows its users to reject (planning) decisions, and to extend and to modify the initial plan.

- Process knowledge managed by the scheduler allows to guide the project members in their activities.

- It integrates process and measurement technologies.

\subsection{Scheduler Support from the User Perspective}

The scheduler as a central component of the new process-sensitive software engineering environment supports different roles in a software development project.

- Project planers are able to define new processes to be performed to reach the project's goal. They may access information about the current state of the project, the reasons which led to it, and dependencies between processes. This information is valuable to change the project plan in the case of an undesired situation (e.g. product changes, new requirements from the customer).

- The Scheduler enables project managers to delegate processes to their team members and to supervise their enactment. The Scheduler automatically notifies team members project changes and therefore reduces the coordination effort of the manager.

- For team members the Scheduler provides a To-Do agenda. When a team member accepts a process waiting for execution, the Scheduler generates a work context which guides him in his activities and allows him to access relevant products and tools. The Scheduler informs him about product modifications which are relevant for his work and notifies him if the process is removed (by the project planner) from the current project plan.

In the process model, we describe only the responsibility for a process by relating process performer conditions to it. The role of the performer can be deduced from what he does during enactment: If he defines a new method or selects a (complex) method, he refines the plan and can be called project planer. Taking the role of a manager, he delegates processes and supervises their enactment. If he produces the outputs of the process, he can be called a team member. Depending on the model every person may assume these three roles, perhaps all at once. So, we are able to describe hierarchical organization structures as well as flexible project structures following the lean management approach.

\subsection{Operationalizing the Project Plans}

The most important entities managed by the scheduler are processes, methods, decisions for methods and products together with their states.

\section{Processes}

During execution a process passes through various states. Every state is determined by the activities done by the process performers and the dependencies to other processes and decisions. State changes happen on ground of process performer activities. A process is managed by the Scheduler as soon as it becomes part of the actual plan.

Entry criteria. A process is enabled as long as all entry criteria are valid. Only an enabled process can be accepted and enacted. 
Delegation. Within a process definition an agent binding is specified. During execution, this binding is evaluated to obtain the set of agents permitted to execute the process. The set is determined by matching the agent bindings with the abilities of the agents. The resulting set of authorized agents is further reduced by the project manager. He delegates the process to a subset of potential process performer.

Agent bindings and delegations can change. If an agent binding or delegation changes before the process has been accepted, the set of authorized agents is adapted. If a delegation changes after a performer has accepted the task, the new performer takes over the role of the old one without changing the process state.

Method selection and rejection. In order to reach the process goal, the project planner selects an applicable method. In Section 7 the Implementation Process knows the two methods Implementation with Structural Testing and Implementation with Functional Testing. The set of applicable methods is a subset of the methods defined in the project plan. This set may be reduced depending on the current project context. Selecting a method results in a decision. A valid decision is part of the actual project plan. The decision for a method can be rejected later. Such a change activity has consequences on other parts of the project because of dependencies between processes, methods and products. Additionally, the method set can be modified by adding or removing methods from the process specification.

Process invariants. The process invariants have to stay valid during the process performance. If they become invalid, the project planer has change the current project plan.

Exit criteria. After the work on a process is finished, its exit criteria are checked. If this checking fails an exception event is forwarded to the process manager who has to resolve this conflict, for example by replanning the process.

\section{Methods}

Applying complex methods. A complex method refines a process into a set of one or more subprocesses. Applying a complex method, each subprocesses is instantiated $x$-fold. The $x$ is determined by the cardinality of the subprocess. In the model, each subprocess has a definite or $\infty$ cardinality. The cardinality $\infty$ is replaced during process execution by a definite value. The consumed, produced and modified product parameters of processes with a cardinality greater one are identified by a definite index.

Applying atomic methods. Atomic methods produce products. In the project plan the name and type of the products that have to be produced are specified. If an atomic method is applied, the resulting products are assigned to the corresponding parameter. Additionally, a dependency between the decision for the method and the produced products is established: the rejection of the decision results in the retraction of the parameter assignments.

Product mapping of complex methods. The product mapping of complex methods allows to exchange products between the superprocess and its subprocesses. In Figure 7 one can see, that product desdoc.sw_des_descr is mapped to product sw_des_descr. The mapping direction is given by the direction of the arrow sign. We distinguish three kinds of mapping:

1. Mapping consumed products. If a consumed product of the superprocess is assigned to the corresponding parameter, the product is also assigned to the parameter of the subprocess, specified in the mapping rule. Therefore the product becomes automatically available for the subprocess.

2. Mapping produced products. If within a method application of a subprocess a product is produced, it is assigned to parameter of the higher level process as specified in the mapping rule.

3. Mapping modified products. If within a subprocess a product is modified, the modified product is assigned to parameter of the higher level process as specified in the mapping rule.

Product interface relations. The product interface relations specify the exchange of products between the subprocesses of a complex method. If a product parameter pair is specified in the interface relations, the product assigned to one of the parameters is automatically assigned to the other one, too. 
Agent binding The agent binding of methods is a subset of those specified in the corresponding process. Again, the set of attributes process performer and process supporters have to fulfill to be authorized to work on the process is matched with the abilities of the agents (see Processes). This semantic leads to the effect, that an agent who accepts a task may only apply a subset of the specified applicable methods.

\section{Dependencies between Processes and Subprocesses}

Complex methods decompose processes in a set of subprocesses. If an agent decides to apply a complex method the subprocesses related to the method becomes part of the actual project plan and have to be solved.

Therefore the validity of the subprocesses depends on the decision for the corresponding complex method. If the decision for the method is rejected, the rationale for the validity of the resulting subtasks is no longer given. The subtasks have to become invalid. Decisions, which have been taken within the subtasks, must be retracted, too.

\section{Product Flow Dependencies}

As described above a product assignment is dependent from decision for the corresponding atomic method. The products produced by an atomic method enter in further decisions and constitute dependencies. The dependencies become important for the process execution, if product producing decisions are rejected. For details see [11,27].

\section{Blocked Process Goals}

Applying a complex method, a process is refined into a set of subprocesses. Every subprocess has to be solved by applying a method. If the set of applicable methods for one ore more subprocesses is empty, their process goal can't be reached. This state is called blocked. In this situation activities to solve this deadlock have to be started.

\subsection{Discussion of the Requirements}

MILOS is based on the MVP-L approach. Therefore, the main concepts of software development processes are supported (R1). The MILOS Scheduler supports project planers and managers (R2, R3). Extending, modifying, and changing the current project plan is supported by the MILOS Scheduler using techniques from CoMo-Kit. Planning and enactment decisions are handled by the Scheduler (R5). Because the dependencies point to the cause of an event, the Scheduler is able to guide the user in reacting on changes appropriately (R6). Alternating planning and enactment steps is supported (R4). MILOS provides an object-centred product model and the Scheduler is able to store products (R7). Process and product attributes and the attribute mappings support measurement activities (R8). By using a workflow engine, the Scheduler, team members are guided and coordinated in their activities. Activities for reacting on events may itself have global effects on the project planning and execution. Because the Scheduler manages dependencies, the performer is relieved from coordination activities resulting from such changes (R9, 10). The MILOS language is designated to automate process steps by using process programs (R11). Currently, this feature is not implemented by the Scheduler but it will be a future extension.

\section{Related Work}

To validate the completeness of our integration we compared it against existing frameworks and definitions that were developed by Conradi, Fernström and Fuggetta [9], Feiler and Humphrey [13], Lonchamp [21], and Armitage and Kellner [2] (see Table A.1 of the appendix). Every framework provides a consistent set of concepts that embodies a particular understanding about aspects of software processes. In contrast to the concepts presented in this paper, aspects of the meta-process (i.e., the process of process modeling) are also described in some of the works $[9,13,21]$. The four definition frameworks are not formalized but nat- 
ural language is used to explain the meaning of concepts. Because the terms were developed in different contexts, one cannot assume a perfect match between them. Therefore, we see the terms from different frameworks as similar, not as equal. Under this assumption, MILOS implements most of the concepts covered by the other frameworks. A predefined type classifying all components of the delivered product (i.e., as proposed by Lonchamp) are not present in either approach. All other abstract concepts covered by the frameworks are considered in MILOS.

Process-sensitive software engineering environments which support evolution of enacted process models are a focal point of current research, but the results are still immature [24, 34]. In the remainder of this section we discuss environments relevant for the work presented in this paper and point out the main differences to our approach. Important requirements not met by the related approaches are checked (which leaves open whether the other requirements are met). The unsatisfied requirements are marked by a ' $\neg$ '.

The SPADE environment is a system for developing analyzing, and enacting process models described in the language SLANG (Spade LANGuage) [3]. Activities are modules with well-defined interfaces and a Petri net specification as a body. Activity types may be changed during enaction but they do not affect existing instances. When the type of an active process is modified, SPADE prompts the user to provide a transformation function. This is a solution for the problem P2 (i.e., type-state correspondence) which is not solved by our approach. SLANG provides only a small set of software development process concepts $(\neg \mathrm{R} 1)$. Also the user must decide when to start process evolution. The system does not provide any support to decide which parts need to be changed ( $\neg \mathrm{R} 5)$.

GRAPPLE is an operator-based approach which supports planning and plan recognition [17]. The operators encapsulate the functionality of both tools and processes performed by agents. Reason maintenance techniques are used to manage dependencies between process steps; dependencies between products are not maintained $(\neg R 5)$. Our synthesized approach extends these techniques by explicitly representing dependencies between products, so that a goal-directed reaction on changes of the product state is possible. GRAPPLE does not allow for alternating planning and enactment $(\neg R 4)$.

The database-oriented EPOS Process Modeling System distinguishes between classes (templates), instances thereof, and information about the creation, change, and conversion of classes and instances on a meta-level [19]. Feedback about correctness and performance of the enacted process model triggers changes of classes and instances which are under version control. Classes and instances may be changed in the case of inactive processes. The user is responsible for establishing consistency between classes and instances. Thus the EPOS Process Modeling System tackles the problems P1 and P2. No dependencies between process fragments are managed ( $\neg$ R5) so it is not possible to determine what processes accessed a faulty product and might be enacted another time. Detection of deviations and recognition of a change's impact are completely left to the user $(\neg \mathrm{R} 6)$.

Redoing is an operation in the Hierarchical and Functional Software Process (HFSP) approach that allows cancellation of erroneous activities and doing that part of the process again [35]. Software development processes are understood as functions organized in a hierarchy (called an enaction tree). Redoing means cutting a subtree out of the enaction tree and replacing it with another tree which is newly enacted. The decision to redo is specified in the process models. It can be seen as a sort of "goto" where results in the subtree are discarded. In contrast to our approach, short-term planning is not supported $(\neg R 3)$, the process models must be completely defined before interpretation $(\neg \mathrm{R} 4)$, and the decisions for redoing are predefined, which means that criteria to detect deviations from the plan must be specified within the models $(\neg \mathrm{R} 5)$.

\section{Summary}

This paper presents the integration of two approaches, namely CoMo-Kit and MVP-E. They both were developed completely independently to solve particular and isolated problems of automated process support. A recent comparison revealed commonalities and differences of both systems [37]. Requirements were set up for process-sensitive software engineering environments. They are addressed by the newly cre- 
ated approach MILOS which is a synthesis of CoMo-Kit and MVP-E. The concepts of MILOS were illustrated by using a scenario of a standard implementation process. Relating MILOS to other works on the one hand the uniqueness of this approach was shown but on the other hand potential future work was identified by pointing to solutions of problems not tackled by MILOS.

Roughly spoken, as an intermediate result of the synthesis MVP-L's concepts are used to describe software processes and the concepts of the CoMo-Kit process engine are used to enact the models. Using these basic elements, we are able to alternate modeling, planning, and enaction modes. Our integrated approach provides a sufficient set of concepts to capture real-world processes. By integrating knowledge based techniques a flexible process-sensitive software engineering environment will be created which manages dependencies between project information and supports backtracking.

Acknowledgments: Sigrid Goldmann did careful work comparing CoMo-Kit and MVP-L. The authors would like to thank the members of both working groups who contributed to many ideas presented in this paper.

\section{References}

[1] P. Armenise, S. Bandinelli, C. Ghezzi, and A. Morzenti. Software process languages: Survey and assessment. In Proceedings of the Fourth Conference on Software Engineering and Knowledge Engineering, Capri, Italy, June 1992.

[2] James W. Armitage and Marc I. Kellner. A conceptual schema for process definitions and models. In Dewayne E. Perry, editor, Proceedings of the Third International Conference on the Software Process, pages 153-165. IEEE Computer Society Press, October 1994.

[3] Sergio C. Bandinelli, Alfonso Fuggetta, and Carlo Ghezzi. Software process model evolution in the SPADE environment. IEEE Transactions on Software Engineering, 19(12):1128-1144, December 1993.

[4] Victor R. Basili, Gianluigi Caldiera, and H. Dieter Rombach. Experience Factory. In John J. Marciniak, editor, Encyclopedia of Software Engineering, volume 1, pages 469-476. John Wiley \& Sons, 1994.

[5] Victor R. Basili, Gianluigi Caldiera, and H. Dieter Rombach. Goal Question Metric Paradigm. In John J. Marciniak, editor, Encyclopedia of Software Engineering, volume 1, pages 528-532. John Wiley \& Sons, 1994.

[6] Victor R. Basili and H. Dieter Rombach. The TAME Project: Towards improvement-oriented software environments. IEEE Transactions on Software Engineering, SE-14(6):758-773, June 1988.

[7] Israel Z. Ben-Shaul, Gail E. Kaiser, and George T. Heineman. An architecture for multi-user software development environments. In H. Weber, editor, Proceedings of the Fifth ACM SIGSOFT/SIGPLAN Symposium on Software Development Environments, pages 149-158, 1992. Appeared as ACM SIGSOFT Software Engineering Notes 17(5), December 1992.

[8] Alfred Bröckers, Christopher M. Lott, H. Dieter Rombach, and Martin Verlage. MVP-L language report version 2. Technical Report 265/95, Department of Computer Science, University of Kaisers-lautern, 67653 Kaiserslautern, Germany, 1995.

[9] Reidar Conradi, Christer Fernström, and Alfonso Fuggetta. A conceptual framework for evolving software processes. ACM SIGSOFT Software Engineering Notes, 18(4):26-35, October 1993.

[10] Bill Curtis, Marc I. Kellner, and Jim Over. Process modeling. Communications of the ACM, 35(9):75-90, September 1992.

[11] Barbara Dellen, Kirstin Kohler, and Frank Maurer. Design rationales and software process models. SFB-Bericht SFB501-01-95, Fachbereich Informatik, Universität Kaisers-lautern, 67653 Kaisers-lautern, November 1995.

[12] Barbara Dellen and Frank Maurer. Integrating planning and execution in software development processes. In Proceedings of the Workshop on Enabling Technologies: Infrastructure for Collaborative Enterprises (WET ICE '96). IEEE CS Press, June 1996. (to appear).

[13] Peter H. Feiler and Watts S. Humphrey. Software process development and enactment: Concepts and definitions. In Proceedings of the Second International Conference on the Software Process, pages 28-40. IEEE Computer Society Press, February 1993. 
[14] Christer Fernström. Process WEAVER: Adding process support to UNIX. In Proceedings of the Second International Conference on the Software Process, pages 12-26. IEEE Computer Society Press, February 1993.

[15] Pankaj K. Garg and Mehdi Jazayeri. Process-centered Software Engineering Environments. IEEE Computer Society Press, 1996.

[16] D. Georgakopoulos, M. Hornick, and A. Sheth. An overview of workflow management: From process modeling to workflow automation infrastructure. Distributed \& Parallel Databases, 3:119-153, 1995. Kluwer Academic Press, Boston.

[17] Karen Erickson Huff. Plan-Based Intelligent Assistance: An Approach to Support the Software Development Process. PhD thesis, University of Massachusetts, September 1989.

[18] Institute of Electrical and Electronics Engineers. IEEE Standard for Developing Software Life Cycle Processes, 1992. IEEE Std. 1074-1991.

[19] M. Letizia Jaccheri and Reidar Conradi. Techniques for process model evolution in EPOS. IEEE Transactions on Software Engineering, 19(12):1145-1156, December 1993.

[20] C. D. Klingler, M. Neviaser, A. Marmor-Squires, C. M. Lott, and H. D. Rombach. A case study in process representation using MVP-L. In Proceedings of the Seventh Annual Conference on Computer Assurance (COMPASS 92), pages 137-146, June 1992 .

[21] Jaques Lonchamp. A structured conceptual and terminological framework for software process engineering. In Proceedings of the Second International Conference on the Software Process, pages 41-53. IEEE Computer Society Press, February 1993.

[22] Christopher M. Lott. Measurement support in software engineering environments. International Journal of Software Engineering \& Knowledge Engineering, 4(3):409-426, September 1994.

[23] Christopher M. Lott, Barbara Hoisl, and H. Dieter Rombach. The use of roles and measurement to enact project plans in MVP-S. In W. Schäfer, editor, Proceedings of the Fourth European Workshop on Software Process Technology, pages 30-48, Noordwijkerhout, The Netherlands, April 1995. Lecture Notes in Computer Science Nr. 913, Springer-Verlag.

[24] Nazim H. Madhavji and Maria H. Penedo. Guest editor's introduction. IEEE Transactions on Software Engineering, 19(12):1125-1127, December 1993. Special Section on the Evolution of Software Processes.

[25] Frank Maurer. Hypermedia-based Knowledge Engineering for distributed, knowledge-based Systems. PhD thesis, Universität Kaiserslautern, 1993. in German.

[26] Frank Maurer. Project coordination in design processes. In Proceedings of the Workshop on Enabling Technologies: Infrastructure for Collaborative Enterprises (WET ICE '96). IEEE CS Press, June 1996. (to appear).

[27] Frank Maurer and Jürgen Paulokat. Operationalizing conceptual models based on a model of dependencies. In A. Cohn, editor, Proceedings of the 11th European Conference on Artificial Intelligence. John Wiley \& Sons, Ltd., 1994.

[28] Frank Maurer and Gerhard Pews. Supporting cooperative work in urban land-use planning. In Proceedings of COOP-96, 1996. (to appear).

[29] Andreas Oberweis. Workflow management in software engineering projects. In S. Medhat, editor, Proceedings of the 2nd International Conference on Concurrent Engineering and Electronic Design Automation, 1994.

[30] Ch. Petrie. Planning and Replanning with Reason Maintenance. PhD thesis, University of Texas, Austin, 1991.

[31] Charles Petrie. Context maintenance. In Proceedings of the AAAI-91, 1991.

[32] H. Dieter Rombach and Martin Verlage. How to assess a software process modeling formalism from a project member's point of view. In Proceedings of the Second International Conference on the Software Process, pages 147-158, February 1993.

[33] H. Dieter Rombach and Martin Verlage. Directions in software process research. In Marvin V. Zelkowitz, editor, Advances in Computers, vol. 41, pages 1-63. Academic Press, 1995. 
[34] Stanley M. Sutton, Jr., Dennis Heimbigner, and Leon J. Osterweil. Language constructs for managing change in process-centered environments. In Proceedings of the Fourth ACM SIGSOFT/SIGPLAN Symposium on Practical Software Development Environments, pages 206-217, 1990. Appeared as ACM SIGSOFT Software Engineering Notes 15(6), December 1990.

[35] Masato Suzuki, Atsushi Iwai, and Takuya Katayama. A formal model of re-execution in software process. In Leon J. Osterweil, editor, Proceedings of the 2nd International Conference on the Software Process, pages 84-99. IEEE, IEEE CS Press, February 1993.

[36] Martin Verlage. Multi-view modeling of software processes. In Brian C. Warboys, editor, Proceedings of the Third European Workshop on Software Process Technology, pages 123-127, Grenoble, France, 1994. Nr. 772, Springer-Verlag.

[37] Martin Verlage, Barbara Dellen, Frank Maurer, and Jürgen Münch. A synthesis of two process support aproaches. In Proceedings of the 8th Software Engineering and Knowledge Engineering Conference (SEKE'96). Knowledge Engineering Institute, June 1996. 


\section{Appendix}

Table A.1 relates software process terms defined by different authors. The terms explain real-world concepts. The definitions given in $[2,9,13,21]$ are in natural language and therefore lack formality. The overview presented in Table A.1 should not be understood as a precise comparison of terminology. The terms differ slightly in their meanings even when they have the same name. The matching was performed based on careful but subjective assessment. The reader is referred to the cited literature for a detailed explanation of the terms. A table cell two high means that the term corresponds to two terms of another framework. Empty cells mean that no term with an equivalent meaning to other terms of that row is defined in the approach discussed in the column.

\begin{tabular}{|c|c|c|c|c|c|c|}
\hline MILOS & CoMo-Kit & MVP-L & $\begin{array}{l}\text { Armitage and } \\
\text { Kellner [2] }\end{array}$ & $\begin{array}{l}\text { Feiler, } \\
\text { Humphrey [13] }\end{array}$ & $\begin{array}{l}\text { Lonchamp } \\
{[21]^{\mathrm{a}}}\end{array}$ & $\begin{array}{l}\text { Conradi et al. } \\
\text { [9] }^{\mathrm{a}}\end{array}$ \\
\hline Process & Task & Process & Process (instance) & Process (Element) & $\begin{array}{ll}\begin{array}{l}\text { Software } \\
\text { cess }\end{array} & \text { Pro- } \\
\end{array}$ & Process \\
\hline \multirow[t]{2}{*}{ Process Type } & & Process Model & Process (type) & Process Definition & & Template \\
\hline & & & & & & Production Process \\
\hline \multirow[t]{3}{*}{ Method } & \multirow[t]{2}{*}{ Method } & & Activity & & Process Step & Activity \\
\hline & & & $\begin{array}{l}\text { Activity } \\
\text { Description }\end{array}$ & Process Script & & \\
\hline & & & Procedure & Process Program & & \\
\hline \multirow[t]{2}{*}{ Atomic Method } & Atomic Method & $\begin{array}{ll}\begin{array}{l}\text { Elementary } \\
\text { cess }^{\mathrm{b}}\end{array} & \text { Pro- } \\
\end{array}$ & & Process Step & Activity & \\
\hline & & & & Task & Task & \\
\hline Process Attribute & & Process Attribute & Activity State ${ }^{\mathrm{c}}$ & & & \\
\hline Complex Method & Complex Method & Refinement & Decomposition & & & \\
\hline $\begin{array}{l}\text { Criteria: } \\
\text { Precondition } \\
\text { Invariant } \\
\text { Postcondition }\end{array}$ & & Criteria & $\begin{array}{l}\text { Behavioral } \\
\text { Information }\end{array}$ & Process Constraint & Constraint & \\
\hline \multirow[t]{2}{*}{ Project Plan } & & \multirow[t]{2}{*}{ Project Plan } & & Process Plan & & \\
\hline & & & & Project Plan & & Software Project \\
\hline \multirow{2}{*}{$\begin{array}{l}\text { Product } \\
\text { (Instance) }\end{array}$} & \multirow[t]{2}{*}{ Concept Instance } & \multirow[t]{2}{*}{ Product } & \multirow[t]{2}{*}{ Artifact (instance) } & & \multirow[t]{2}{*}{ Artifact } & Artifact (Input) \\
\hline & & & & & & $\begin{array}{l}\text { Software Item (Out- } \\
\text { put) }\end{array}$ \\
\hline Product Type & Concept Class & Product Model & Artifact (type) & & & Template \\
\hline \multirow[t]{3}{*}{ Product Slot } & Attribute & Elementary Product & & & & \\
\hline & & & & & & Software Product \\
\hline & & & & & Deliverable & \\
\hline Product Attribute & & Product Attribute & Artifact Statec & & & \\
\hline Product Flow & Information flow & Product Flow & Artifact Flow & & & \\
\hline Ressource & & Resource & & & Resource & \\
\hline Agent & Agent & Personnel & Agent & \multirow[t]{2}{*}{ Agent } & \multirow[t]{2}{*}{ Agent } & Agent \\
\hline Tool & & Tool & & & & Tool \\
\hline Property & & Resource Attribute & Agent Statec & & & \\
\hline \multirow[t]{2}{*}{ Attribute } & & Attribute & Attribute $^{\mathrm{d}}$ & & & \\
\hline & & Model & Process Definition & Process Definition & $\begin{array}{l}\text { Generic Process } \\
\text { Model }\end{array}$ & Template \\
\hline
\end{tabular}

\section{Table A.1: Relating Different Frameworks}

a.Not all process terms presented in $[9,13,21]$ are considered in the corresponding columns, because many of them describe no concept of software development processes but ideas of enaction (e.g., enactment state), organizational processes (e.g., monitoring), or process characteristics (e.g., liveness).

b.Process which contains no refinement

c.Predefined attributes used by a process engine (interpretation machine) to manage an overall project state.

d.Attribute is explained as "a textual description of information". This general and abstract definition matches the other terms in the row only partially. 


\section{The Sonderforschungsbereich 501}

The "Deutsche Forschungsgemeinschaft (DFG)" is a major sponsor of basic research activities in Germany. Besides individual projects DFG sponsors long-term strategic research activities at German universities. The most prestigious form of funding are so-called "Sonderforschungsbereiche (SFBs)", special research institutes aimed at addressing fundamental research areas. Specific characteristics of SFBs include their affiliation with a highly respected scientific department at a German university, funding periods of 9 to 15 years (with regular evaluations), and interdisciplinary collaboration.

The SFB 501 on "Development of Large Systems with Generic Methods" was started at the University of Kaiserslautern on January 01, 1995. It aims at developing and evaluating a set of techniques, methods and tools for supporting the fast and reliable customization of complex domain specific software systems. The emphasis is on techniques, methods and tools that support reuse of all kinds of software artifacts ranging from system components to process fragments and other related knowledge.

In the first step existing techniques, methods and tools are being evaluated for suitability within the domain of process control - starting with the application scope of building automation.

The mid-term goal of the SFB is to generalize the resulting techniques, methods and tools such that they can be used within other application scopes and domains to establish similar reuse-based development processes.

The long-term goal of the SFB is to contribute to the science base for transforming software development from an art to an engineering discipline.

Within the SFB several research groups from the departments of Computer Science and Electrical Engineering collaborate. Current employment count includes 8 professors, 17 full-time researchers and about the same number of part-time student assistants. The following projects have been established:

- Application scope "Building Automation": A project to investigate requirements on such systems and build simulation models for system/acceptance testing

- Software Engineering Laboratory: A project to support both the prototype development of a first building automation system and the evaluation of experiments

- Experiment-Based modeling of software development processes/knowledge based planning and control of SE processes: Two projects to support the planning and execution of processes for software development and experimentation based on existing knowledge

- Generic communication systems/Generic system software: Two projects to investigate the possibilities for generic modeling of system software needed within the chosen application domain

- Formal description techniques: A project to select, modify, integrate and evaluate the appropriate description techniques for generic development

Additional projects will be proposed at the next SFB review. 